\title{
Clinical Investigation: The Presence of Viral Meningitis without Pleocytosis among Pediatric Patients
}

\author{
Adi Klein-Kremer ${ }^{1}$, Vered Nir $^{1}$, Kassem Eias ${ }^{1}$, Rony-Reuven Nir ${ }^{1}$, Renata Yakubov ${ }^{1}$, \\ Keren Gershon ${ }^{2}$ \\ ${ }^{1}$ Department of Pediatrics, Hillel-Yaffe Medical Center, Hadera, Israel \\ ${ }^{2}$ Unit of Infectious Diseases, Hillel-Yaffe Medical Center, Hadera, Israel \\ Email: ${ }^{*}$ adi@hy.health.gov.il
}

Received 17 September 2014; revised 14 October 2104; accepted 10 November 2014

Copyright (C) 2014 by authors and Scientific Research Publishing Inc.

This work is licensed under the Creative Commons Attribution International License (CC BY). http://creativecommons.org/licenses/by/4.0/

(c) (i) Open Access

\section{Abstract}

Background: Viral meningitis (VM) is mostly common among infants. Its induction by enteroviruses (EVM) is associated with morbidity and is primarily diagnosed by lumbar puncture, which may yield false negatives. We evaluated the frequency of VM by polymerase chain reaction (PCR) among infants with no detected pleocytosis. Furthermore, as literature suggests EVM essentially occurs during summer and fall, we characterized the monthly distribution of EVM cases. Methods: Infants diagnosed with VM from June 2009 to May 2010 were enrolled in the current prospective study. Following each lumbar puncture, CSF was tested for enteroviruses by PCR. Outcome measures were i) the percentage of EVM cases revealed solely by PCR; and ii) the monthly distribution of EVM cases. Results: Enrolled were 173 VM-diagnosed infants, of whom $75(43.4 \%)$ tested positive in CSF-PCR. Of these, no pleocytosis was indicated in $43(57 \%)$, specifically in $70 \%$ and $42 \%$ of infants younger than 90 days and older than 1 year, respectively. Furthermore, $119(69 \%)$ infants were admitted during June-November while 54 (31\%) during December-May. Conclusions: Current findings stress the high frequency of infants who were tested negative for pleocytosis, yet were diagnosed with VM by PCR. This was especially noticeable among infants younger than 3 months, possibly reflecting their decreased ability to mount a robust inflammatory response to EV infection. CSF-PCR may be warranted in pediatric patients who test negative for pleocytosis. While most EVM cases occurred during the summer and fall, EVM-infants were admitted to the hospital all through the year.

\section{Keywords}

Cerebrospinal Fluid (CSF), Enteroviral Meningitis, Lumbar Puncture, Polymerase Chain Reaction (PCR), Viral Meningitis

\footnotetext{
${ }^{*}$ Corresponding author.
}

How to cite this paper: Klein-Kremer, A., Nir, V., Eias, K., Nir, R.-R., Yakubov, R. and Gershon, K. (2014) Clinical Investigation: The Presence of Viral Meningitis without Pleocytosis among Pediatric Patients. Open Journal of Pediatrics, 4, $276-282$. http://dx.doi.org/10.4236/ojped.2014.44038 


\section{Introduction}

Viral meningitis (VM) is a syndrome characterized by acute onset of meningeal symptoms and fever, with pleocytosis of the cerebrospinal fluid (CSF) and no growth on routine bacterial culture [1]. Although VM can occur at any age, it is most frequently reported in infants younger than one year [2] [3]. Importantly, many VM cases undoubtedly go unreported [2] [4] [5].

Enteroviruses account for most viral meningitis cases-across all ages-in which the cause is recognized [2]-[4] [6]. The term "enteroviruses" addresses the transmission mode rather than the infection symptoms; indeed, infections with these ubiquitous viruses are typically asymptomatic [7]. However, enteroviruses may induce systemic infections, and are prone to be neuroinvasive; almost all enteroviruses types can give rise to neurological manifestations ranging from VM to meningoencephalitis and paralytic poliomyelitis [7]. Enteroviral typing is essential for identifying and monitoring outbreaks [8]. Infants and young children with impaired immunity are most susceptible to enteroviruses, and the incidence decreases with age [6]. Infection is seasonal in temperate climates with most reports in summer and autumn [6].

Most cases that present clinically with meningitis are self-limiting and carry a good prognosis. Nevertheless, enteroviral meningitis (EVM) causes morbidity, with moderate or high fever despite anti-pyretics and several days of severe headache warranting opiate analgesia [9]. Abrupt deterioration in mental status or seizures may be caused by progression from meningitis to meningoencephalitis [6]. Over the past 2 decades, vaccination policies, the HIV epidemic, altered sexual behavior and increasing travel have altered the spectrum of causative EVM agents [7]. No specific antiviral treatment is available, and management is conservative; immunoglobulin replacement has a role in patients with hypogammaglobulinaemia who are prone to severe and chronic enteroviral disease [7].

A pivotal methodology for EVM diagnosis includes lumbar puncture, performed to collect a CSF sample that is tested for pleocytosis (increase of white blood cells in the CSF). In case of a positive indication, the CSF sample is tested for enteroviruses by polymerase chain reaction (PCR). Importantly, lumbar puncture, which is believed to be crucial in EVM diagnosis [7], has been associated with a considerable amount of false negatives [10]. This may partially account for the sizable extent of VM cases that are unreported [2] [4] [5]. Conversely, CSF-PCR, estimated to be threefold to 1000-fold more sensitive than routine viral culture [7] [11], may yield a higher diagnostic accuracy for EVM. Furthermore, CSF-PCR may be utilized for the identification of the specific viral cause, which is clinically advantageous for informing prognosis, improving patient care, lessening antibiotics use, decreasing hospital length of stay and serving to avert further infection spread [7].

The current prospective study aimed at evaluating the percentage of EVM cases reported negative for pleocytosis by lumbar puncture yet positive for enteroviruses using PCR. This will elucidate the clinical importance of the CSF-PCR test in diagnosing pediatric patients with EVM who tested negative for pleocytosis. The current study is especially relevant since classic clinical diagnostic signs for meningitis have much variability and thus limited value in children [12]. Furthermore, we assessed the monthly distribution of EVM cases during a time period of one year.

\section{Methods}

Infants diagnosed with VM at Hillel-Yaffe Medical Center Department of Pediatrics from June 2009 to May 2010 were enrolled in the current prospective study. The institutional ethics committee approved the current study, which was performed in accordance to the Helsinki Declaration. A signed consent form was collected from the children's parents.

\subsection{Lumbar Puncture}

Lumbar puncture was performed by inserting a spinal needle between the lumbar vertebrae L3/L4 or L4/L5 until cerebral fluid was leaking. The fluid was cultured for bacteriae, checked for i) cell count; ii) glucose; and iii) protein content, and tested for enteroviruses and herpes by PCR.

\subsection{PCR Assays of CSF}

\subsubsection{Viral RNA Isolation}

An aliquot of the samples $(200 \mu \mathrm{l})$ was used to extract viral RNA using the MagNA Pure LC Total Nucleic Acid Isolation Kit (Roche Diagnostics, Basel, Switzerland) as described previously [13]. Each sample was eluted in 
$50 \mu$ buffer. All samples had been spiked before extraction with an internal control virus (phocine distemper virus) to monitor for efficient extraction and amplification, as described previously [14].

\subsubsection{RT-qPCR}

The isolated viral RNA was reverse transcribed using Multi-Scribe RT and random hexamers (Applied Biosystems, Foster City, CA). Detection of EV as performed using a RT-qPCR assay as previously described [15]. In addition, an in-house RT-qPCR assay was developed for the detection of HPeV using Primer Express (Applied Biosystems). Conserved target regions were identified using BLAST (www.ncbi.nlm.nih.gov/blast). Sequences of the primers and probes used have been summarized by de Crom et al., 2012 [16]. Potential cross-reactivity of the $\mathrm{HPeV}$-pecific assay with other related picornaviruses was excluded by using the RT-qPCR assay on samples known to contain RNA of Coxsackievirus A (A2, A9, A13, A14, A16, A24), Coxsackievirus B (B1, B3, B4, B5), Echovirus (1-7, 9, 12, 14, 15, 17, 19-21, 24-27, 29, 31, 32), Enterovirus 71, Poliovirus 1, Poliovirus 3 and/or Rhinoviruses. Real-time PCR procedures were performed as described previously [13].

\subsection{Statistical Analysis}

All analyses were conducted using SPSS (Statistical Package for the Social Sciences), Version 19 (SPSS Inc., Chicago, IL, USA). Statistical significance was set at $\mathrm{P}<0.05$. Z-scores were used to test differences in proportions of two samples. Outcome measures were i) the percentage of EVM cases revealed solely by PCR; and ii) the monthly distribution of EVM cases.

\section{Results}

Enrolled were 173 VM-diagnosed infants, of whom 96 (55.5\%) were males and 77 (44.5\%) were females. A total of 100 (58\%) infants were Jewish, while 73 (42\%) were Arab.

Of the entire study cohort, 75 infants (43.4\%) tested positive in CSF-PCR tests, while 98 (56.6\%) tested negative. Of the former group of 75 patients, lumbar puncture indicated pleocytosis only in 32 (42.6\%) infants. No pleocytosis was indicated in the remaining majority of 43 (57.3\%) infants.

Figure 1 describes the number of VM- and EVM-diagnosed infants, both by PCR and lumbar puncture, categorized by the age groups of $0-1$ month, 1 - 3 months, 3 - 12 months, and $>12$ months. Figure 2 depicts the proportions of the EVM-diagnosed infants by each method within each age group. Of the 173 admitted VM-diagnosed infants, 104 (60\%) were 0 - 3 months old. Of these, 47 were EVM-diagnosed by PCR. A majority of 33 of these infants $(70 \%)$ tested negative for pleocytosis by the lumbar puncture test, and had been therefore initially ruled out as suffering from EVM. Of the 173 admitted VM-diagnosed infants, 48 (28\%) were $>12$ months old. Of these, 19 were EVM-diagnosed by PCR. Eight of these infants (42\%) tested negative for pleocytosis by the lumbar puncture test, and had been therefore initially ruled out as suffering from EVM.

Figure 3 presents the monthly distribution of VM- and EVM-diagnosed infants, both by PCR and lumbar

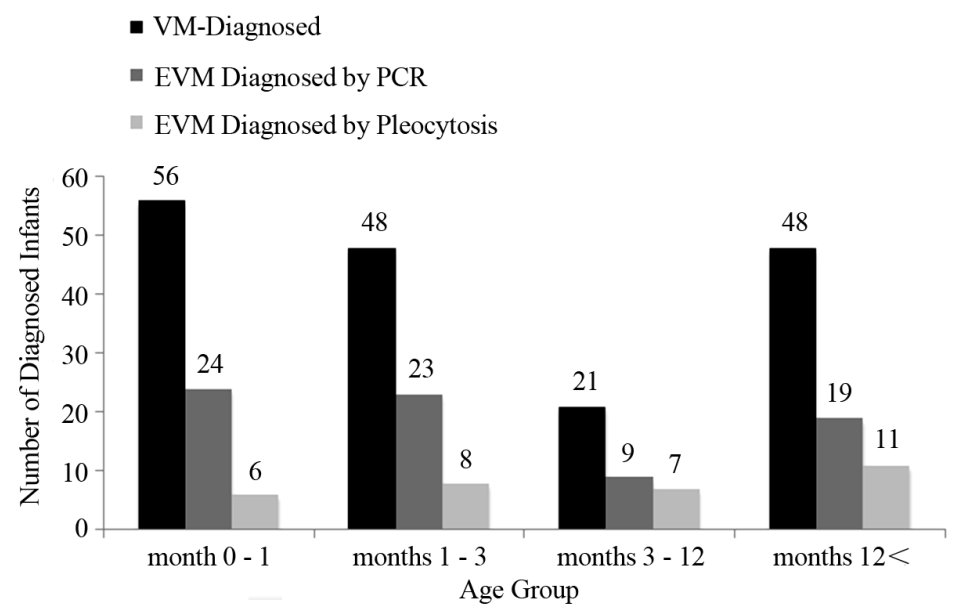

Figure 1. The number of VM- and EVM-diagnosed infants, both by PCR and lumbar puncture, categorized by age groups. 
- (\%) EVM Diagnosed by PCR

(\%) EVM Diagnosed by Pleocytosis

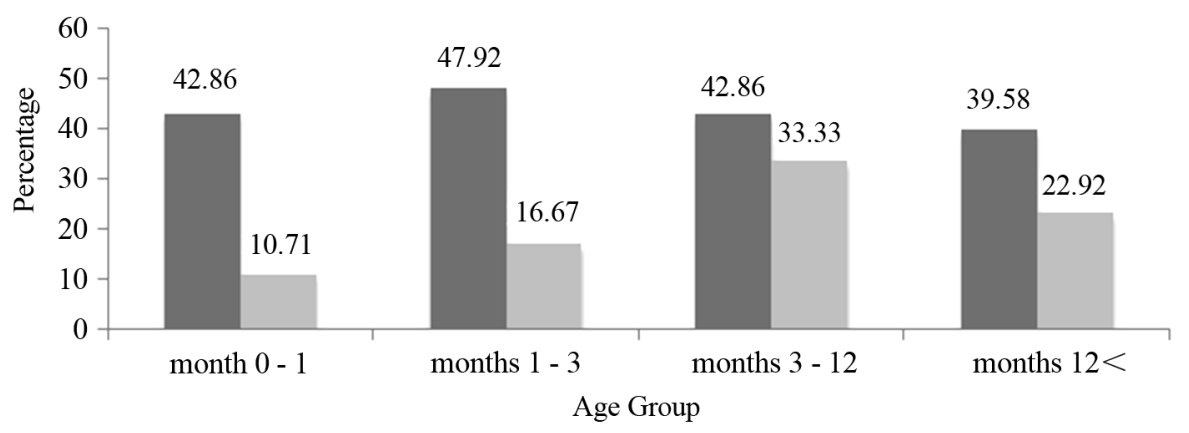

Figure 2. The proportions of the EVM-diagnosed infants by the PCR and lumbar puncture methods, categorized by age group.

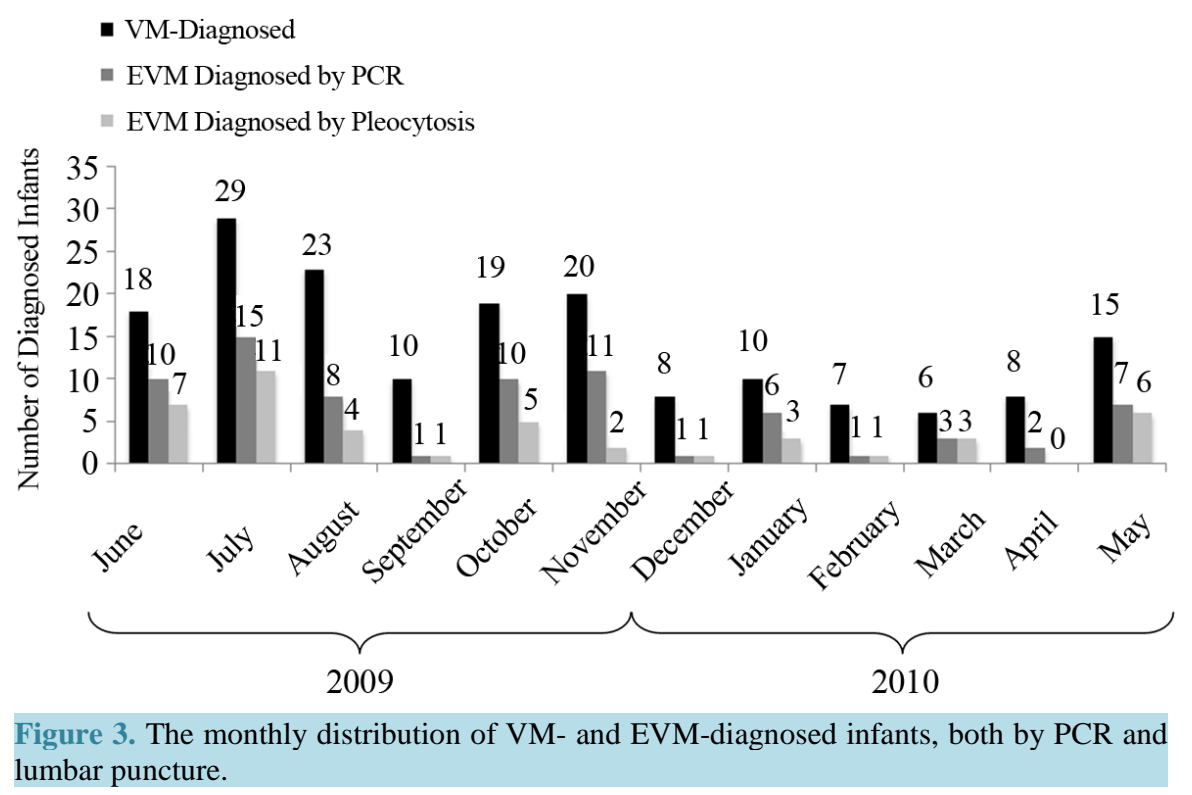

puncture. During the summer-fall months of June-November, 119 (69\%) infants were admitted due to VM, of whom 55 (46.2\%) were diagnosed with EVM. During the winter-spring months of December-May, the corresponding number of EVM cases was 54 (31\%), of whom 25 (46.3\%) were diagnosed with EVM. While the observed proportion of VM admissions was significantly greater during the summer-fall months compared to the winter-summer months $(\mathrm{P}<0.001)$, the proportions of EVM cases during each of these periods were comparable.

\section{Discussion}

The main finding of the current study is that $57.3 \%$ of the infants who were tested positive for VM by PCR tested negative for pleocytosis by lumbar puncture and had been therefore initially ruled out as suffering from EVM. Specifically, as many as $70 \%$ of the infants younger than 3 months tested negative for pleocytosis, while the corresponding percentage among infants older than 12 months was $42 \%$. Hence, the occurrence of VM false negatives, as indicated by the lumbar puncture test, was evident in the entire study sample, although chiefly manifested among infants younger than 3 months. This may attest to the lowered capacity of younger infants to mount a robust inflammatory response to EV infection.

Current findings suggest that CSF-PCR may be warranted in pediatric patients who test negative for pleocytosis, especially in infants younger than 3 months. This aligns with the study by Mulford et al. [17], who re- 
ported that 30\% of EVM-diagnosed infants younger than 3 months demonstrated no pleocytosis; the corresponding percentage among those aged 2 months - 18 years was $2 \%$. This supports the view that among infants with EV infections, the absence of CSF pleocytosis is related to younger age and lower peripheral WBC counts, probably reflecting the decreased ability of younger infants to robustly respond to EV infection [18]. In keeping, Gomez et al. [19] reported that among 65 infants younger than 3 months diagnosed with EVM, pleocytosis was detected only in 26 (40\%). Accordingly, a reliable examination such as the CSF-PCR is in principal clinical need. This notion may be further supported by the fact that pertinent biomarkers have proven to be ineffective in EVM prediction; Gomez et al. [19] also found that the symptoms in the investigated infants with EVM were similar to those in infants with a self-limited febrile process without intracranial infection, and that C-reactive protein (CRP) and WBC count were not associated with EVM diagnosis.

Existing literature indicates that the CSF enterovirus PCR test may improve the care of infants potentially suffering from VM, not only for its accurate diagnosis [20]-[23]; based on 276 pediatric patients, Ramers et al. [20] reported that positive EVM-PCR results promoted rapid discharge of patients. King et al. [21] collected data for 478 infants younger than 90 days and found that having positive CSF enterovirus PCR results decreased hospitalization length and the duration of antibiotic use. In the same line, Dewan et al. [22] suggested that among febrile infants 56 days or younger, a positive CSF enterovirus PCR test result was associated with a shorter hospital length of stay compared with untested infants.

In a comprehensive study, Archimbaud et al. [24] found that i) 63\% of children with EVM detected by CSFPCR were discharged immediately (mean: 2.5 hours) and 95\% within 24 hours; and ii) PCR results allowed discontinuation of antibiotics in $50 \%-60 \%$ of all patients treated. Clinical data were compared between patients whose positive CSF-PCR results were available within 24 hours and those whose results were available in more than 24 hours after collection of CSF. While the duration of antibiotic treatment was shorter in the former group, no difference was observed in the length of stay. Therefore, while it may be advantageous to finalize the CSF tests within 24 hours, delayed results are still clinically gainful for the patients.

As to the demonstrated monthly distribution of EVM, previous reports indicate that higher EVM incidence may be associated with warm months [19] [25]. Meehan and Bachur [25] have investigated 2003 febrile infants, of whom 176 (8.8\%) had a CSF pleocytosis; they reported that pleocytosis risk during the nonsummer months was $5 \%$, while during the summer season it increased to $17.4 \%$. Current findings suggest that while the observed percentage of VM admissions was found to be greater during the summer-fall months, as compared to the winter-spring months, the proportions of EVM cases during each of these periods were comparable. This implies that EVM risk—among Israeli infants—may not be seasonally affected, but rather, equally spread throughout the year.

\section{Conclusion}

In conclusion, current findings add an important stratum of evidence to previous reports by demonstrating the exceedingly high percentage of young infants who tested negative for pleocytosis, yet were diagnosed with EVM. This points to the paramount clinical need in a reliable test for EVM diagnosis, without which epidemiologic reports of VM cases will indisputably remain fallible [2] [4] [5]. We recommend that EVM-PCR assays are routinely tested as part of the opening management of meningitis, specifically among infants in whom no pleocytosis was detected.

\section{References}

[1] United States Department of Health and Human Services Centers for Disease Control and Prevention. Aseptic Meningitis 1990 Case Definition. www.cdc.gov/epo/dphsi/casedef/asmeningitiscurrent

[2] Rantakallio, P., Leskinen, M. and Von Wendt, L. (1986) Incidence and Prognosis of Central Nervous System Infections in a Birth Cohort of 12,000 Children. Scandinavian Journal of Infectious Diseases, 18, 287-294. http://dx.doi.org/10.3109/00365548609032339

[3] Kupila, L., Vuorinen, T., Vainionpää, R., Hukkanen, V., Marttila, R.J. and Kotilainen, P. (2006) Etiology of Aseptic Meningitis and Encephalitis in an Adult Population. Neurology, 66, 75-80. http://dx.doi.org/10.1212/01.wnl.0000191407.81333.00

[4] Davison, K.L. and Ramsay, M.E. (2003) The Epidemiology of Acute Meningitis in Children in England and Wales. Archives of Disease in Childhood, 88, 662-664. http://dx.doi.org/10.1136/adc.88.8.662

[5] Health Protection Agency. Diseases Notifiable (to Local Authority Proper Officers) under the Public Health (Infectious 
Diseases) Regulations 1988. www.hpa.org.uk/infections/topics_az/noids/noidlist.htm

[6] Sawyer, M.H. and Rotbart, H. (2004) Viral Meningitis and Aseptic Meningitis Syndrome. In: Scheld, W.M., Whitley, R.J. and Marra, C.M., Eds., Infections of the Central Nervous System, 3rd Edition, Lippincott Williams \& Wilkins, Philadelphia, 75-93.

[7] Logan, S.A.E. and MacMahon, E. (2008) Viral Meningitis. BMJ, 336, 36-40. http://dx.doi.org/10.1136/bmj.39409.673657.AE

[8] Pallansch, M.A. and Roos, R.P. (2001) Enteroviruses: Polioviruses, Coxsackieviruses, Echoviruses, and Newer Enteroviruses. In: Knipe, D.M., Howley, P.M., Griffin, D.E., Lamb, R.A., Martin, M.A., Roizman, B., et al., Eds., Fields Virology, 4th Edition, Lippincott Williams \& Wilkins, Philadelphia, 723-775.

[9] Rotbart, H.A., Brennan, P.J., Fife, K.H., et al. (1998) Enterovirus Meningitis in Adults. Clinical Infectious Diseases, 27, 896-898. http://dx.doi.org/10.1086/517162

[10] Read, S.J. and Kurtz, J.B. (1999) Laboratory Diagnosis of Common Viral Infections of the Central Nervous System by Using a Single Multiplex PCR Screening Assay. Journal of Clinical Microbiology, 37, 1352-1355.

[11] Thomas, J.E., Falls, E., Velasco, M.E. and Zaher, A. (2000) Diagnostic Value of Immunocytochemistry in Leptomeningeal Tumor Dissemination. Archives of Pathology \& Laboratory Medicine, 124, 759-761.

[12] Amarilyo, G., Alper, A., Ben-Tov, A. and Grisaru-Soen, G. (2011) Diagnostic Accuracy of Clinical Symptoms and Signs in Children with Meningitis. Pediatric Emergency Care, 27, 196-199. http://dx.doi.org/10.1097/PEC.0b013e31820d6543

[13] van de Pol, A.C., Wolfs, T.F., Jansen, N.J., van Loon, A.M. and Rossen, J.W. (2006) Diagnostic Value of Real-Time Polymerase Chain Reaction to Detect Viruses in Young Children Admitted to the Paediatric Intensive Care Unit with Lower Respiratory Tract Infection. Critical Care, 10, R61. http://dx.doi.org/10.1186/cc4895

[14] van Doornum, G.J., Guldemeester, J., Osterhaus, A.D. and Niesters, H.G. (2003) Diagnosing Herpesvirus Infections by Real-Time Amplification and Rapid Culture. Journal of Clinical Microbiology, 41, 576-580. http://dx.doi.org/10.1128/JCM.41.2.576-580.2003

[15] Nijhuis, M., van Maarseveen, N., Schuurman, R., et al. (2002) Rapid and Sensitive Routine Detection of All Members of the Genus Enterovirus in Different Clinical Specimens by Real-Time PCR. Journal of Clinical Microbiology, 40, 3666-3670. http://dx.doi.org/10.1128/JCM.40.10.3666-3670.2002

[16] de Crom, S.C., Obihara, C.C., van Loon, A.M., et al. (2012) Detection of Enterovirus RNA in Cerebrospinal Fluid: Comparison of Two Molecular Assays. Journal of Virological Methods, 179, 104-107. http://dx.doi.org/10.1016/j.jviromet.2011.10.007

[17] Mulford, W.S., Buller, R.S., Arens, M.Q. and Storch, G.A. (2004) Correlation of Cerebrospinal Fluid (CSF) Cell Counts and Elevated CSF Protein Levels with Enterovirus Reverse Transcription-PCR Results in Pediatric and Adult Patients. Journal of Clinical Microbiology, 42, 4199-4203. http://dx.doi.org/10.1128/JCM.42.9.4199-4203.2004

[18] Seiden, J.A., Zorc, J.J., Hodinka, R.L. and Shah, S.S. (2010) Lack of Cerebrospinal Fluid Pleocytosis in Young Infants with Enterovirus Infections of the Central Nervous System. Pediatric Emergency Care, 26, 77-81. http://dx.doi.org/10.1097/PEC.0b013e3181ce2fad

[19] Gomez, B., Mintegi, S., Rubio, M.C., Garcia, D., Garcia, S. and Benito, J. (2012) Clinical and Analytical Characteristics and Short-Term Evolution of Enteroviral Meningitis in Young Infants Presenting with Fever without Source. Pediatric Emergency Care, 28, 518-523. http://dx.doi.org/10.1097/PEC.0b013e3182587d47

[20] Ramers, C., Billman, G., Hartin, M., Ho, S. and Sawyer, M.H. (2000) Impact of a Diagnostic Cerebrospinal Fluid Enterovirus Polymerase Chain Reaction Test on Patient Management. JAMA, 283, 2680-2685. http://dx.doi.org/10.1001/jama.283.20.2680

[21] King, R.L., Lorch, S.A., Cohen, D.M., Hodinka, R.L., Cohn, K.A. and Shah, S.S. (2007) Routine Cerebrospinal Fluid Enterovirus Polymerase Chain Reaction Testing Reduces Hospitalization and Antibiotic Use for Infants 90 Days of Age or Younger. Pediatrics, 120, 489-496. http://dx.doi.org/10.1542/peds.2007-0252

[22] Dewan, M., Zorc, J.J., Hodinka, R.L. and Shah, S.S. (2010) Cerebrospinal Fluid Enterovirus Testing in Infants 56 Days or Younger. Archives of Pediatrics and Adolescent Medicine, 164, 824-830. http://dx.doi.org/10.1001/archpediatrics.2010.153

[23] Graham, A.K. and Murdoch, D.R. (2005) Association between Cerebrospinal Fluid Pleocytosis and Enteroviral Meningitis. Journal of Clinical Microbiology, 43, 1491. http://dx.doi.org/10.1128/JCM.43.3.1491.2005

[24] Archimbaud, C., Chambon, M., Bailly, J.L., et al. (2009) Impact of Rapid Enterovirus Molecular Diagnosis on the Management of Infants, Children, and Adults with Aseptic Meningitis. Journal of Medical Virology, 81, 42-48. http://dx.doi.org/10.1002/jmv.21330

[25] Meehan 3rd, W.P. and Bachur, R.G. (2008) Predictors of Cerebrospinal Fluid Pleocytosis in Febrile Infants Aged 0 to 90 Days. Pediatric Emergency Care, 24, 287-293. http://dx.doi.org/10.1097/PEC.0b013e31816ecbb0 
A. Klein-Kremer et al.

\section{Acronyms}

VM: Viral meningitis

EVM: Enteroviral meningitis

CSF: Cerebrospinal fluid

PCR: Polymerase chain reaction 
Scientific Research Publishing (SCIRP) is one of the largest Open Access journal publishers. It is currently publishing more than 200 open access, online, peer-reviewed journals covering a wide range of academic disciplines. SCIRP serves the worldwide academic communities and contributes to the progress and application of science with its publication.

Other selected journals from SCIRP are listed as below. Submit your manuscript to us via either submit@scirp.org or Online Submission Portal.
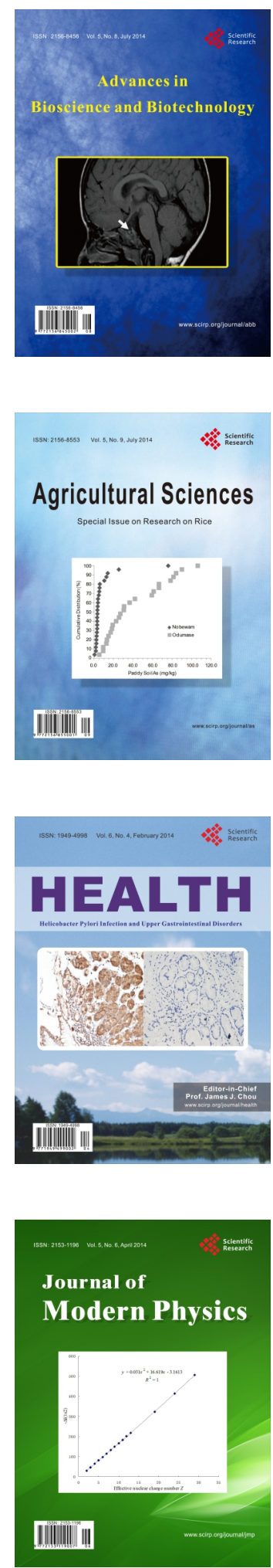
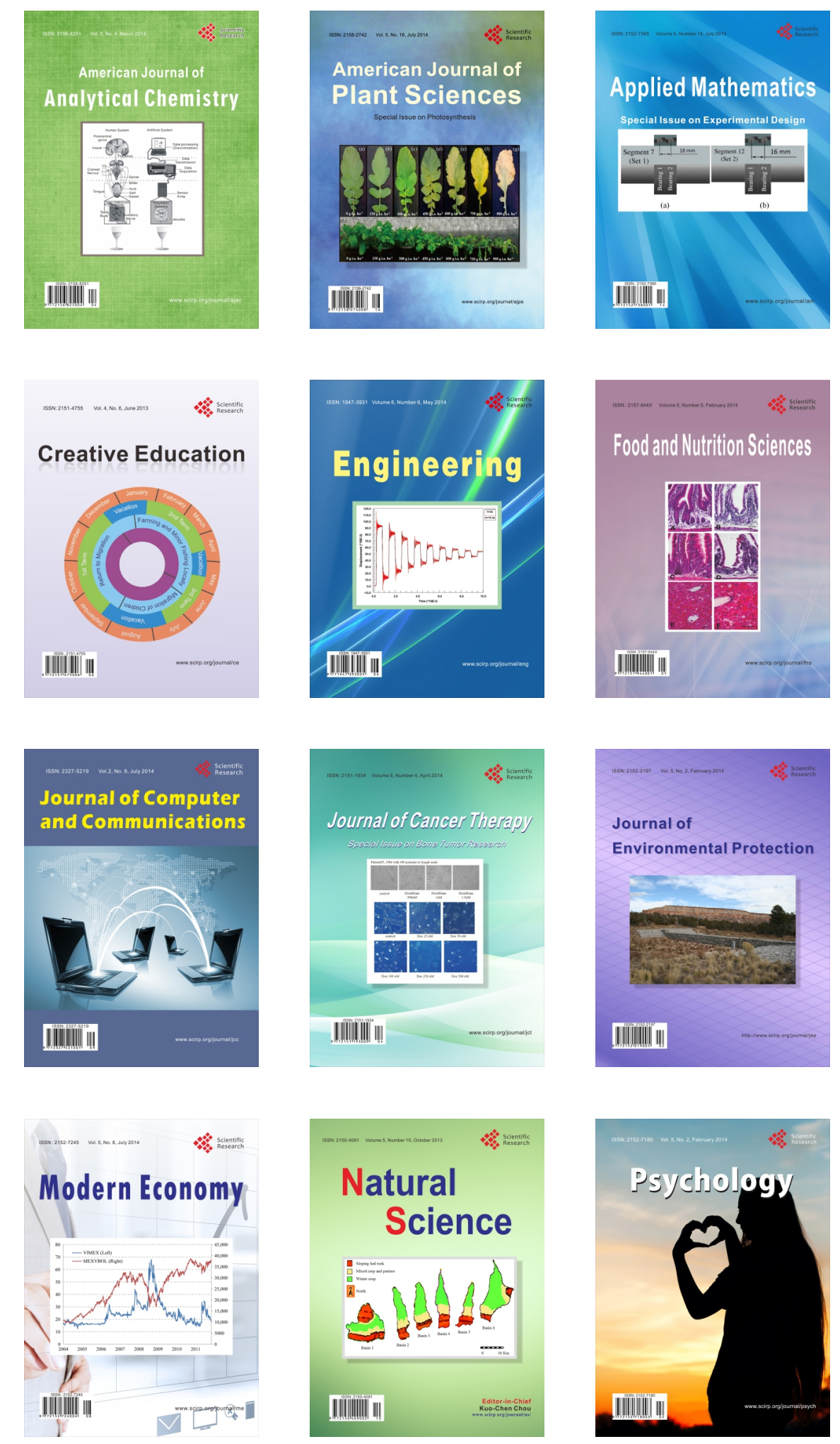